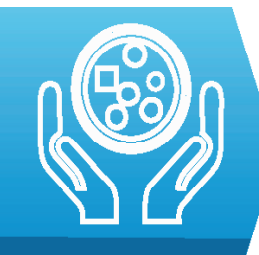

ONCOLOGY
Department of Pathology, ABVIMS (formerly PGIMER), Dr RML Hospital, New Delhi, India

\section{Pure yolk sac tumor of testis with lung metastasis in an adult patient - case report}

\author{
Pushpanjali Behera, Arvind Ahuja, Minakshi Bhardwaj
}

\begin{abstract}
Pure yolk sac tumor is the most frequently seen testicular tumor in childhood; however it is usually a component of mixed germ cell tumor in adults. Pure yolk sac tumors are quite rare in adults. Pure yolk sac tumor in adults has been categorized as yolk sac tumor, post-pubertal type. We report a case of pure yolk sac tumor of right testis with lung metastasis in a 37-year-old man who was successfully treated by high inguinal orchidectomy and BEP combination chemotherapy.
\end{abstract}

Keywords: pure yolk sac tumor, germ cell tumor, metastasis, chemotherapy, adult, endodermal sinus tumor

\section{Introduction}

Testicular tumors comprise $1 \%$ of male neoplasms and 5\% of urological tumors, with 3-10 new cases occurring per 100,000 males/per year in Western countries [1]. Testicular yolk sac tumors (YST) are a type of non-seminomatous germ cell tumor. In the recent WHO 2016 classification of germ cell tumors of the testis, YST can be of pre pubertal type or post pubertal type. Prepubertal YSTs occur in infants and young children; they are not associated with germ cell neoplasia in situ (GCNIS), and almost always occur in the pure form [2]. While post pubertal type yolk sac tumors are GCNIS associated tumors and occur in adults. They are of more concern, especially if they are seen in pure form.

Pure YST of the testis in an adult is extremely rare [3]. In adults it exhibits a more aggressive behavior with a high frequency of metastasis compared to childhood YST [4].

\section{Case report}

A 37-year-old male presented with slowly increasing swelling and pain over right testis for the past 6-7 years. On examination right testis was enlarged, measuring about $12 \times 10 \times 8 \mathrm{~cm}$, but localized to the scrotum. It was soft on palpation at the base but firm at the upper pole. Cough impulse was absent and transillumination test was negative. Left testis was normal in size and consistency. Ultrasonography (USG) of the scrotum revealed diffuse heterogeneous echogenicity with evidence of multiple hypoechoic areas and internal calcification along with increased vascularity. On imaging the differential diagnosis of neoplastic or chronic infective etiology were suggested. Abdominal USG, in addition, showed hepatomegaly and retroperitoneal lymph nodes. Contrast enhanced computed tomography (CECT) abdomen revealed enhancing heterogeneous right testicular mass lesion with hydrocele and thickened right spermatic cord (Figure 1A). CECT of chest showed a solitary nodule in the anterior segment of left upper lobe suggesting metastasis from the testicular mass (Figure 1B). Biochemical profile showed serum alpha-fetoprotein (AFP) markedly elevated $3120 \mathrm{ng} / \mathrm{ml}$, elevated serum lactate dehydrogenase (LDH) 484 $\mathrm{U} / 1$ and $\beta$-human chorionic gonadotropin $(\beta-h C G)$ was within normal limits [1.5 mIU/l]. Hematological parameters revealed mild anemia ( $\mathrm{Hb} 11.9 \mathrm{gm} / \mathrm{dl})$ otherwise within normal limits. 


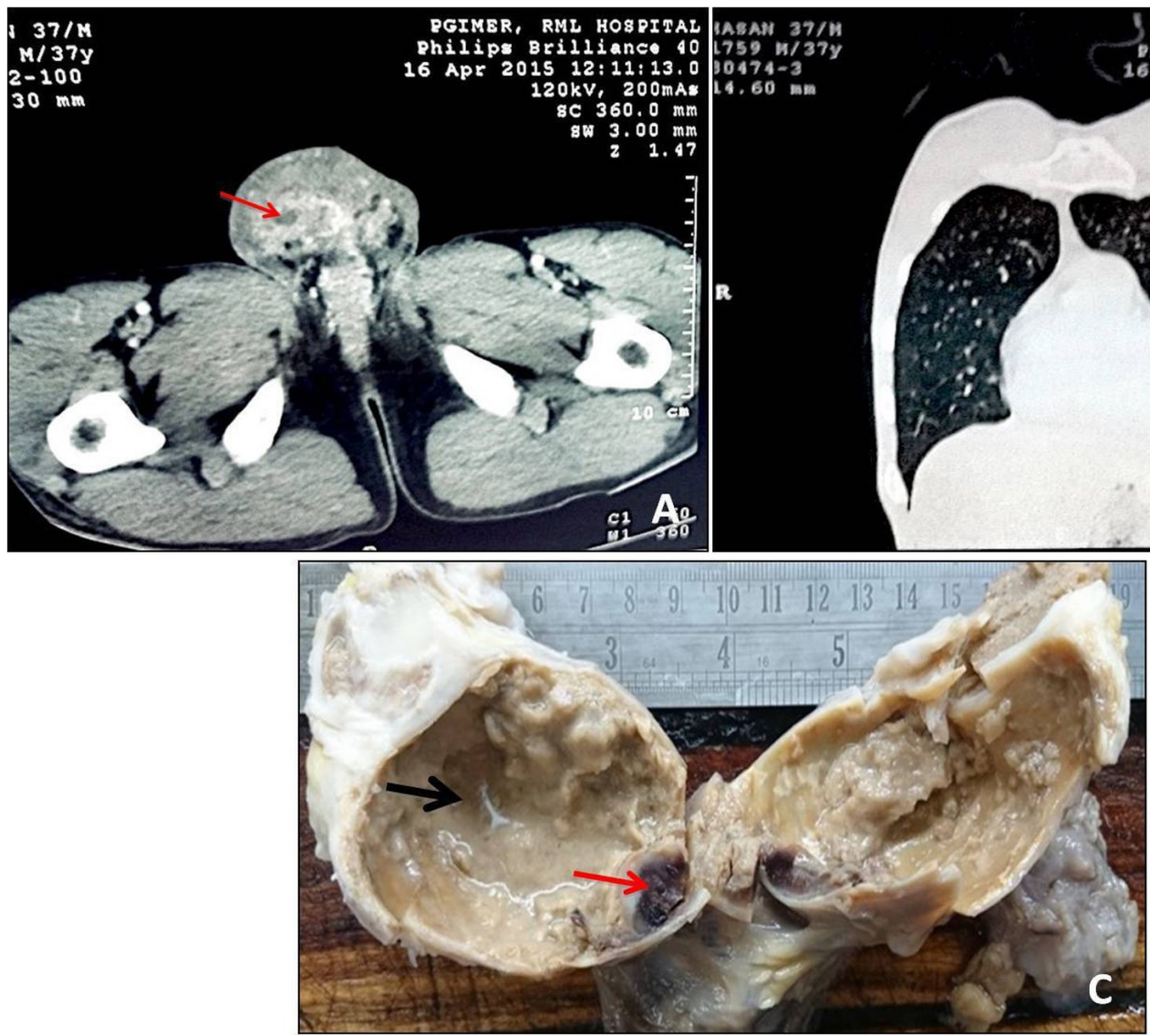

Figure 1 A-C. A- CECT image showing an infiltrating heterogenous enhancing mass lesion in the right testis; B- CECT lung showing metastatic solitary nodule in left upper lobe; C- Gross of orchidectomy specimen showing grey white tumor along with areas of hemorrhage (red arrow) and necrosis (black arrow).

Subsequently, the patient underwent high inguinal orchidectomy. On histopathological examination, grossly the right testis was enlarged measuring $10 \times 9 \times 8 \mathrm{~cm}$ and attached spermatic cord measured $4 \mathrm{~cm}$ in length. External surface was smooth glistening with congested blood vessels without any breach of tunica. On serial slicing the testicular parenchyma was completely replaced by well circumscribed grey white tumor with variegated appearance (Figure 1C). Large areas of necrosis and focal hemorrhage were noted. Multiple sections were taken, which on microscopy showed a well circumscribed tumor. The tumor showed predominantly endodermal sinus pattern, focal solid sheets and reticular or microcystic pattern (Figure 2A-C). Many Schiller Duval bodies were noted (Figure 2B). Focally PAS positive diastase resistant hyaline globules were also noted (Figure 2D). Tumor cells were mild to moderately pleomorphic with round vesicular nucleus, conspicuous nucleoli and scant amount of eosinophilic to clear cytoplasm. Brisk mitosis was noted. Large areas of necrosis and focal hemorrhage were also seen. Tumor emboli were present in some of the vessels. Few atrophic seminiferous tubules were identified at the periphery in one of the sections examined. Extensive sampling was done to rule out other germ cell tumor components. Based on characteristic histomorphological features a diagnosis of pure yolk sac tumor was made. A panel of immunohistochemical (IHC) stain was applied for the confirmation of the diagnosis and to rule out mixed germ cell tumor. The tumor cells showed diffuse positivity for pancytokeratin (Figure 2F) and focally for AFP (Figure 2E). However, C-kit (CD117), PLAP, CD30 and CEA were negative.

Following histopathological diagnosis, 4 cycles of BEP combination chemotherapy regimen was given to the patient, which was completed within 3 months. At this point, the patient was tested for serum AFP and LDH levels, which came out as $55.6 \mathrm{ng} / \mathrm{ml}$ and $250 \mathrm{U} / \mathrm{I}$ respectively. Patient was followed at regular intervals. After 2 months of completion of chemotherapy, a follow-up CECT chest showed absence of the pulmonary nodule as seen earlier in left upper lobe, in the pre-operative CT scan. At 4-year follow up, the patient is alive and doing well without comorbidities or any evidence of relapse. His serum AFP level is $1.94 \mathrm{IU} / \mathrm{ml}$ (normal range $<5.8 \mathrm{IU} / \mathrm{ml}$ ) and chest $\mathrm{CT}$ is normal. 

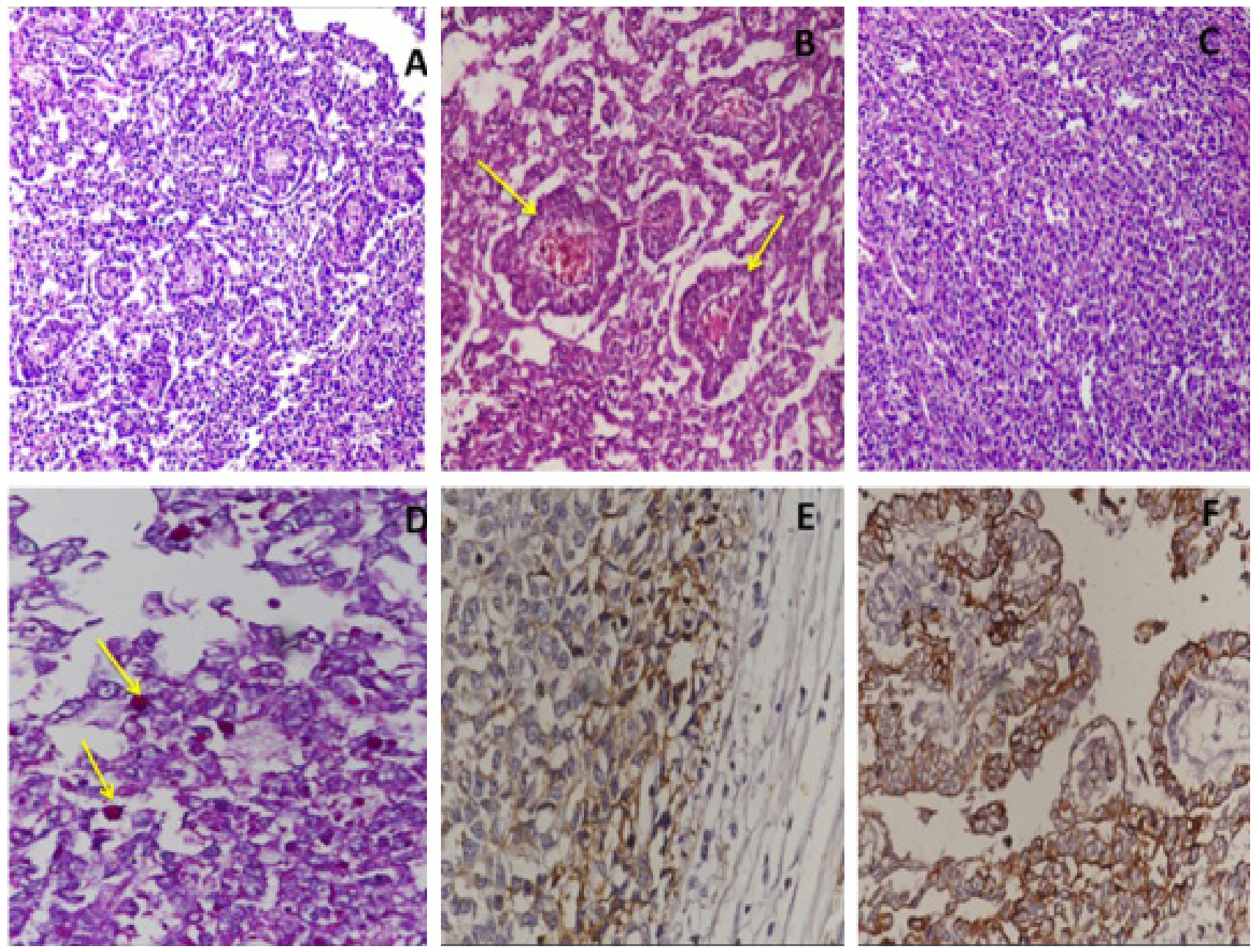

Figure 2 A-F. Photomicrograph of tumor. A- endodermal sinus pattern [H\&E, 200X]; B- Schiller Duval body (yellow arrows) [H\&E, 400X]; C- solid sheet; D- PAS positive hyaline globules (yellow arrows) [H\&E, 400X]; E and F- Tumor cell were focally immunoreactive for AFP [E] and revealed diffuse Pan-CK [F] positivity [IHC, 400X].

\section{Discussion}

YST or endodermal sinus tumor is characterized by several patterns that recapitulate the embryonic yolk sac, allantois and extra embryonic mesenchyme. In the testis YST is seen in two distinct age groups - infants, young children and postpubertal males. In children, it is the most common testicular neoplasm accounting for $80 \%$ of the cases [2]. Pure YST is extremely rare in adults and found in $2.4 \%$ of adult patient [2]. In adults, YST usually occurs as a component of mixed germ cell tumor. Talerman et al. did not found a single case of pure YST amongst 147 germ cell neoplasms of testis in adults which they observed over a $4 \frac{1}{2}$-year period [5]. Wang et al. in their case report and literature review published in 2015 mentioned that less than 20 cases of pure yolk sac tumor in adults have been reported [3]. To the best of our efforts, we could find four more reported cases in literature published in English between 2015 and 2019. Janugade et al. reported a case of pure YST arising from cryptorchid testis in a 46-yearold male, who presented with a large abdominal lump extending from infra umbilical region to pelvis along with delayed bowel movement [6]. Murcia et al. reported a similar case of pure YST in a 20 -year-old male, who also had metastases to the liver, retroperitoneum, and lung [7]. The patient succumbed to illness, just 3 months after diagnosis.

LDH, $\beta$-hCG and AFP are the recommended serum markers and used most commonly for the evaluation of testicular tumors. In the present case, LDH and AFP were elevated, but their levels decreased significantly in the post-operative period and subsequently normalized 1 year after completion of chemotherapy.

Grossly, pure YST are non-encapsulated, solid, gray-white to tan, homogeneous mass with a myxoid or 
gelatinous cut surface. Cystic change may be seen. Large tumors usually show areas of hemorrhage or necrosis. Histopathological features are the same regardless of the patient's age. Several different patterns are usually admixed, and may be present in equal amounts, although not infrequently one pattern may predominate. Reticular (microcystic, vacuolated or honeycomb) is the most common pattern. In our case, predominant pattern was endodermal sinus pattern with many Schiller Duval bodies. The immune profile of YST is quite variable. Positive staining for AFP is helpful in the diagnosis but the reaction is variable and sometimes weak. Negative staining does not exclude a diagnosis of YST. AFP is focally demonstrable in approximately $92 \%$ of YST. PLAP shows variable positivity in $40 \%$ to $85 \%$ of cases.

Prepubertal YST has a greater tendency to metastasize via hematogenous route, bypassing retroperitoneal lymph nodes whereas there is no evidence of such in pure post pubertal YST [8]. Blood borne metastasis occurs most frequently to lungs, liver, brain and bone. Although there are no apparent differences in tumor morphology between the prepubertal and postpubertal forms of YST, the prepubertal type yolk sac tumors behave less aggressively than the postpubertal type tumors, with a significantly lower frequency of relapse on surveillance of clinical stage I patients and of lymphatic-based metastases [4]. Our patient presented in stage III (TNM classification), therefore according to guidelines on testicular cancer 2015 update, BEP combination chemotherapy regimen was given [8]. In case of metastatic non-seminomatous germ cell tumor, the recommended schedule includes physical examination, serum tumor markers, plain $\mathrm{X}$ ray of chest, abdominopelvic $\mathrm{CT}$, chest $\mathrm{CT}$ and brain $\mathrm{CT}$ [8]. Only clinical stage and degree of AFP elevation are of prognostic value. In non-seminomatous tumors, advanced stages (IIC and III) are considered to be poor prognosis when associated with any of the following criteria: visceral metastases, AFP $>10.000 \mathrm{ng} / \mathrm{ml}, \mathrm{HCG}>50.000 \mathrm{IU} / 1$ or $\mathrm{LDH}>10 \times$ ULN [8].
In conclusion, even though pure YSTs are childhood tumors, they can rarely occur in adults. Diagnosis of pure YST in an adult should be rendered only after extensive tissue sampling and vigilant search for any other germ cell component. Our patient presented at an advanced stage with lung metastasis and was successfully treated by combination of high inguinal orchidectomy and chemotherapy. This case highlights that despite of fulfilling all poor prognostic criteria, the patient should be offered standard treatment, as the biological behavior of this tumor might not be always aggressive. Further research in post-pubertal pure YST is warranted for better management of patients.

\section{References}

1. Rosen A, Jayram G, Drazer M, Eggener SE. Global trends in testicular cancer incidence and mortality. Eur Urol. 2011;60:374-379.

2. Khan S, Jetley S, Pujani M, Neogi S. Pure yolk sac tumor of testis in an adult: a rare occurrence. J Postgrad Med. 2014;60:351-353.

3. Wang Z, Yan B, Wei YB, Yin Z, Zhou KQ, Yang JR. Adult metastatic yolk sac tumor descending from an intraabdominal testis: A case report and review of the literature. Oncol Lett. 2015;10:3647-3650.

4. Moch H, Cubilla AL, Humphrey PA, Reuter VE, Ulbright TM. The 2016 WHO Classification of Tumours of the Urinary System and Male Genital Organs-Part A: Renal, Penile, and Testicular Tumours. Eur Urol. 2016;70:93-105.

5. Talerman A. The incidence of yolk sac tumor (endodermal sinus tumor) elements in germ cell tumors of the testis in adults. Cancer. 1975;36:211-215.

6. Janugade H, Monteiro J, Gouda S. Pure yolk sac tumour, post-pubertal type, arising from cryptorchid testes. BMJ Case Rep. 2019;12:e229541. doi:10.1136/bcr-2019-229541.

7. Murcia JDU, Ochoa MAS, Manrique IAV, Herrera JMS. Pure Yolk Sac Testicular Tumor in an Adult Patient: Case Report. Oncol Cancer Case Rep. 2017;3:139.

8. Albers P, Albrecht W, Algaba F, Bokemeyer C, CohnCedermark G, Fizazi K, et al. Guidelines on testicular cancer: 2015 update. Eur Urol. 2015;68:1054-1068. 